\title{
Control of Dengue Vector by the Sterile Insect Technique Considering Logistic Recruitment ${ }^{1}$
}

L. ESTEVA2, Departamento de Matemáticas, Facultad de Ciencias, UNAM e Lab-Epifisma, 04510, México, D.F., México.

H.M. YANG ${ }^{3}$, Departamento de Matemática Aplicada, Instituto de Matemática, Estatística e Computação Científica, UNICAMP e Lab-Epifisma, Cx.P. 6065, 13081-970, Campinas, SP, Brasil.

\begin{abstract}
We propose a mathematical model to assess the effects of irradiated male insects introduction in a previously infested region, taking into account the logistic recruitment of sterile male insects. The release of sterile male insects aims to displace gradually the natural (or wild) insect from the habitat. We discuss the suitability of this release technique when applied to peridomestic adapted Aedes aegypti mosquitoes which are transmitters of Yellow Fever and Dengue disease.
\end{abstract}

\section{Introduction}

E. F. Knipling [7] had conceived an approach to insect control in which the natural reproductive processes of insects are disrupted by the use of mutagens such as gamma radiation thus rendering the insects sterile. These sterile insects are then released into the environment in very large numbers in order to mate with the native insects that are present in the environment. A native female that mates with a sterile male will produce eggs, but the eggs will not hatch. If there is a sufficiently high number of sterile insects then most of the crosses are sterile, and as time goes on, the number of native insects decreases and the ratio of sterile to fertile insects increases, thus driving the native population to extinction, which is now known as the sterile insect release technique (SIT) [2].

In the traditional insecticide control, the amount of pesticide used and subsequent cost of utilizing that pesticide is generally proportional to the area treated and not to the size of the population. However, the damage done by a pest is proportional to the number of individual pest per unit area. Thus, the cost/benefit ratio of insecticidal control increases as the size of the pest population increases. But, in sterile male release programs, the number of sterile male released and their

\footnotetext{
${ }^{1}$ Financial support: CNPq (Edital Universal) and FAPESP (Projeto Temático).

${ }^{2}$ lesteva@lya.fciencias.unam.mx.

${ }^{3}$ hyunyang@ime.unicamp.br, grant from CNPq.
} 
cost is proportional to the size of the population. Since the cost/benefit ratio decreases with a decrease in size of the pest population, it can be wise to integrated insecticidal methods with sterile male technique.

SIT was first used successfully in 1958 in Florida to control Screwworm fly (Cochliomya omnivorax) [8]. About 50 million flies were released per week over an 18 months period, in a total of 2 billion flies over 85,000 square mile area. The pest was eradicated after this period of application, in which 40 tons of ground meat were required each week and 20 aircraft to release the sterile flies. The total cost was about US $\$ 10,000,000.00$. Much research was carried out about 30 years ago, especially in India and El Salvador, on the application of SIT to mosquitoes. Unfortunately this technique virtually ends in the mid-1970s, not because the method was a technical failure, but because of political problems in India and intensifying civil wars in Central America [9]. There is now a revival of interest especially in the use of transgenesis to improve sex separation so that only non biting males are released but to ensure that their female progeny die without the need for radiation or chemosterilization [3]. Therefore, with some improvements through the use of currently available transgenic technologies, SIT could become a mainstay for public health control of specific vector-borne diseases. A number of mathematical models have been done to assist the effectiveness of the SIT (see, e.g., [1] [4] [10]).

In this paper we are concerned about the application of the SIT for the control of Aedes aegypti mosquitoes, which is the principal transmissor of Yellow Fever and Dengue disease. This pest species have more than one life stage, which is known that complicates the outcome of a sterile release program. In order to shed further light on the effectiveness of the technique for control of mosquitoes we propose a general model that incorporates two life stages of the pest population. We consider two issues of mosquito behavior that are specially relevant to SIT, namely, a) mating competitiveness of artificially reared sterilized males; b) dispersal ability to ensure that sterile males are released near enough to all emerging wild females so that they have a fair chance of mating with them.

\section{The Model}

Aiming the description of the dynamics of biological control, the life cycle of an insect is divided in two stages: the immature (eggs, larvae and pupae) and the adult one. In the case of mosquitoes the immature phase occurs in water. We denote by $A$ the population size of the immature phase of the insect at time $t$. For the adult form we consider the following compartments: females before mating (singles), $I$; mating fertilized females, $F$; mating unfertilized females, $U$; and male insects, $M$. The population size of sterile (irradiated or transgenic) insects at time $t$ is denoted by $M_{T}$.

The per capita mortality rates of the immature form, unmating females, mating fertilized females, mating unfertilized females, natural (or wild) and sterile male insects are denoted by $\mu_{A}, \mu_{I}, \mu_{F}, \mu_{U}, \mu_{M}$ and $\mu_{T}$, respectively. The net oviposition rate per female insect is proportional to their density, but it is also regulated by a carrying capacity effect depending on the occupation of the available breeder sites. 
In this model we assume that the per capita oviposition rate is given by $\phi(1-A / C)$ where $C$ is the carrying capacity related to the amount of available nutrients and space, and $\phi$ is the intrinsic oviposition rate. The immature population becomes adult insects at a per capita rate $\gamma$; a proportion $r$ of such are females and $1-r$, males.

Flows from $I$ to $F$ and $U$ compartments depend mainly on the number of encounters of females with native and sterile males, and on the correspondingly mating rates. Here we assume that the probability of an encounter of a female with natural insects is given by $M /\left(M+M_{T}\right)$. Then the per capita rate at which female insects are fertilized is $\beta M /\left(M+M_{T}\right)$, where $\beta$ is the mating rate of natural insects.

Since sterile (irradiated or transgenic) insects are placed artificially, it is natural to think that the probability of an encounter of a sterile male with a female depends not only on the number of such males $\left(M_{T} /\left(M+M_{T}\right)\right)$, but also on how far they are placed from the breeding sites. We will assume that this net probability is given by $p M_{T} /\left(M+M_{T}\right)$, where the parameter $p$, with $0 \leq p \leq 1$, is the proportion of sterile insects that are sprayed in the adequate places. Also, the effective fertilization during the mating could be diminished due to the sterilization, which leads us to assume that the effective mating rate of sterile insects is given by $q \beta$, with $0 \leq q \leq 1$. Putting together the assumptions above we get that $\beta_{T} M_{T} /\left(M+M_{T}\right)$ is the per capita rate at which female insects are fecundated with sterile sperm, where $\beta_{T}=p q \beta$. In some extent, the parameter $p$ is related to the effectiveness of sterile male introduction regarded to the spatial distribution of female insects, while $q$ can be thought as a physiological modification induced by the sterilization technique. The parameter $p$ plays an important role in the case of insects whose reproduction depends strongly on the spatially non-homogeneous distribution of breeding sites, like mosquitoes Aedes aegypti.

Similar to the natural population of insects, we assume that the growth rate of sterile male insects is regulated by a carrying capacity effect. This feature takes into account the limiting capacity (of laboratories, for instance) to produce and release sterilized male insects, which allows us to describe the recruitment rate of sterile male insects by $\alpha M_{T}\left(1-M_{T} / K\right)$, where $K$ is the maximum capacity related to the sterile insect production. Note that $\alpha\left(1-M_{T} / K\right)$ is the per capita recruitment rate and here $\alpha$ means the intrinsic recruitment rate.

According to the assumption above the model is given by

$$
\left\{\begin{array}{l}
A^{\prime}=\phi\left(1-\frac{A}{C}\right) F-\left(\gamma+\mu_{A}\right) A \\
I^{\prime}=r \gamma A-\frac{\beta M I}{M+M_{T}}-\frac{\beta_{T} M_{T} I}{M+M_{T}}-\mu_{I} I \\
F^{\prime}=\frac{\beta M I}{M+M_{T}}-\mu_{F} F \\
M^{\prime}=(1-r) \gamma A-\mu_{M} M \\
M_{T}^{\prime}=\alpha M_{T}\left(1-\frac{M_{T}}{K}\right)-\mu_{T} M_{T}
\end{array}\right.
$$


and the remaining decoupled equation for mating unfertilized females is

$$
U^{\prime}=\frac{\beta_{T} M_{T} I}{M+M_{T}}-\mu_{U} U
$$

We proceed with the steady state analysis.

\section{Analysis of the Model}

The system (2.1) for the sterile male insects has two equilibrium points: $\bar{M}_{T_{0}}=0$ and $\bar{M}_{T_{1}}=\left(\alpha-\mu_{T}\right) K / \alpha$. The last one is biologically feasible if and only if $\alpha>\mu_{T}$. Since the sterile male population is governed without the influence of the natural population, the stability analysis of the fifth equation of the system (2.1) alone shows that the trivial equilibrium $\bar{M}_{T_{0}}$ is stable when $\alpha>\mu_{T}$; otherwise, it is unstable and arises the stable non-trivial equilibrium $\bar{M}_{T_{1}}$. This behavior must govern strongly the dynamics of the interaction between natural (wild) and artificially produced populations.

The value $\bar{M}_{T_{0}}=0$ gives rise to two equilibrium points. One is the trivial equilibrium $P_{0}=(0,0,0,0,0)$, plus $\bar{U}=0$, where both natural and sterile male insects are absent, and the other is an equilibrium where only natural insects survive

$$
P_{1}=\left(\bar{A}, \frac{r \gamma}{\beta+\mu_{I}} \bar{A}, \frac{\left(\gamma+\mu_{A}\right) C}{\phi(C-\bar{A})} \bar{A}, \frac{(1-r) \gamma}{\mu_{M}} \bar{A}, 0\right),
$$

where $\bar{A}=\frac{C(R-1)}{R}$ and $R$, which is interpreted below, is given by

$$
R=\frac{\phi r \gamma \beta}{\left(\mu_{A}+\gamma\right)\left(\beta+\mu_{I}\right) \mu_{F}}
$$

Notice that $P_{1}$ is biologically feasible if and only if $R>1$, hence for $R<1$ we have the trivial equilibrium $P_{0}$.

Linear analysis reveals that $-\mu_{M}$ and $\alpha-\mu_{T}$ are eigenvalues of the Jacobian of system (2.1) around $P_{0}$ and $P_{1}$. For $P_{0}$ the other eigenvalues are the roots of the polynomial

$$
\begin{aligned}
& \lambda^{3}+\left(\gamma+\mu_{A}+\beta+\mu_{I}+\mu_{F}\right) \lambda^{2}+\left[\left(\gamma+\mu_{A}\right)\left(\beta+\mu_{I}\right)+\mu_{F}\left(\gamma+\mu_{A}+\beta+\mu_{I}\right)\right] \lambda \\
& +\mu_{F}\left(\gamma+\mu_{A}\right)\left(\beta+\mu_{I}\right)(1-R) .
\end{aligned}
$$

It can be seen readily that the conditions for stability of the above polynomial are satisfied if and only if $R<1$. On the other hand, the other eigenvalues for $P_{1}$ are given by the roots of

$$
\begin{aligned}
& \lambda^{3}+\left[R\left(\gamma+\mu_{A}\right)+\beta+\mu_{I}+\mu_{F}\right] \lambda^{2}+\left[R\left(\gamma+\mu_{A}\right)\left(\beta+\mu_{F}+\mu_{I}\right)+\mu_{F}\left(\beta+\mu_{I}\right)\right] \lambda \\
& +\mu_{F}\left(\gamma+\mu_{A}\right)\left(\beta+\mu_{I}\right)(R-1) .
\end{aligned}
$$

This polynomial is stable if and only if $R>1$. Summarizing, for $\alpha<\mu_{T}$, $P_{0}$ is stable if $R<1$. If $R>1, P_{0}$ becomes unstable and $P_{1}$ emerges as stable equilibrium 
point. This situation represents the absence of any kind of biological control (or a very inefficient control program).

Global stability of the equilibrium point $P_{0}$ can be proved for $R \leq 1 \mathrm{using}$ the function $V: R_{+}^{5} \rightarrow R$ given by

$$
V=\frac{r \gamma}{\mu_{A}+\gamma} A+I+\frac{\phi r \gamma}{\left(\mu_{A}+\gamma\right) \mu_{F}} F
$$

whose orbital derivative

$$
\dot{V}=-\left[\frac{r \gamma \phi}{\left(\mu_{A}+\gamma\right) C}\right] A F-\frac{\left(\beta+\mu_{I}\right)}{M+M_{T}}(1-R) I M-\frac{\left(\beta_{T}+\mu_{I}\right) I M_{T}}{M+M_{T}}
$$

is less or equal to zero for $R \leq 1$. From inspection of system (2.1) it can be seen that the maximal invariant set contained in $\dot{V}=0$ is $P_{0}$. Then, from La-Salle Lyapunov Theorem [6], $P_{0}$ is globally asymptotically stable for $R \leq 1$.

The parameter $R$ can be interpreted biologically as follows: since $\left(\gamma+\mu_{A}\right)^{-1}$ is the average time of survival of the immature phase of the insect, $\gamma^{-1}$ is the average time of its permanence as such, and $r$ is the fraction of immature forms that become females, then $r \gamma\left(\gamma+\mu_{A}\right)^{-1}$ is the probability that an egg will succeed to become a female insect. Arguing in the same way, $\beta\left(\beta+\mu_{I}\right)^{-1}$ is the probability that a female will be fertilized, and finally $\phi \mu_{F}^{-1}$ is the average number of eggs lay by each fertilized female. Thus, the product of these three quantities, which is equal to $R$, is the average number of secondary female insects produced by a single female insect. In demographic terms $R$ is the basic offspring number of insect population (equivalent to basic reproductive number in the epidemiological context). For natural (or wild) insects to maintain in nature, condition $R>1$ is necessary. However, it is reasonable to assume that, in the presence of sterile insects, this could not be a sufficient condition since a proportion of the females are not fertilized.

$$
\begin{aligned}
& \text { Now, for } \bar{M}_{T_{1}}=\frac{\left(\alpha-\mu_{T}\right) K}{\alpha} \text {, the corresponding equilibrium points are } \\
& \qquad \begin{aligned}
& P_{2}=\left(0,0,0,0, \bar{M}_{T_{1}}\right), \\
& P_{3-}=\left(\bar{A}_{-}, \frac{r \gamma \bar{A}_{-}\left[(1-r) \gamma \bar{A}_{-}+\mu_{M} \bar{M}_{T_{1}}\right]}{\left(\mu_{I}+\beta\right)(1-r) \gamma \bar{A}_{-}+\left(\mu_{I}+\beta_{T}\right) \mu_{M} \bar{M}_{T_{1}}}, \frac{\left(\gamma+\mu_{A}\right) C \bar{A}_{-}}{\phi\left(C-\bar{A}_{-}\right)},\right. \\
&\left.\frac{(1-r) \gamma \bar{A}}{\mu_{M}}, \bar{M}_{T_{1}}\right)
\end{aligned}
\end{aligned}
$$

and

$$
\begin{aligned}
P_{3+}= & \left(\bar{A}_{+}, \frac{r \gamma \bar{A}_{+}\left[(1-r) \gamma \bar{A}_{+}+\mu_{M} \bar{M}_{T_{1}}\right]}{\left(\mu_{I}+\beta\right)(1-r) \gamma \bar{A}_{+}+\left(\mu_{I}+\beta_{T}\right) \mu_{M} \bar{M}_{T_{1}}}, \frac{\left(\gamma+\mu_{A}\right) C \bar{A}_{+}}{\phi\left(C-\bar{A}_{+}\right)}\right. \\
& \left.\frac{(1-r) \gamma \bar{A}_{+}}{\mu_{M}}, \bar{M}_{T_{1}}\right)
\end{aligned}
$$

where $\bar{A}_{-}$and $\bar{A}_{+}$are given, respectively, by

$$
\bar{A}_{-}=\frac{(R-1)}{2 R} C\left[1-\sqrt{1-\frac{4 R S}{(R-1)^{2}}}\right]
$$


and

$$
\bar{A}_{+}=\frac{(R-1)}{2 R} C\left[1+\sqrt{1-\frac{4 R S}{(R-1)^{2}}}\right],
$$

which are solutions of the polynomial

$$
p(A)=\frac{R}{C} A^{2}-(R-1) A+C S=0 .
$$

The parameter $R$ is given by the equation (3.1), and $S$ is defined as

$$
S=\frac{\left(\beta_{T}+\mu_{I}\right) \mu_{M}\left(\alpha-\mu_{T}\right) K}{\left(\beta+\mu_{I}\right)(1-r) \gamma C \mu_{T}} .
$$

The remaining decoupled mating unfertilized females is given by

$$
\bar{U}=\frac{\alpha \beta_{T} \mu_{M}}{\mu_{U}\left[\alpha \mu_{M}+A(1-r) \gamma \mu_{T}\right]},
$$

with $A$ being substituted by $\bar{A}_{-}$and $\bar{A}_{+}$to produce the equilibria $P_{3-}$ and $P_{3+}$, respectively.

The parameter $S$ can be written as the ratio between $s_{1}$ and $s_{2}$ where

$$
s_{1}=\left(\beta_{T}+\mu_{I}\right) \frac{r \gamma C}{\mu_{I}} \frac{\left(\alpha-\mu_{T}\right) K}{\mu_{T}} \quad \text { and } \quad s_{2}=\left(\beta+\mu_{I}\right) \frac{r \gamma C}{\mu_{I}} \frac{(1-r) \gamma C}{\mu_{M}} .
$$

The quantities $s_{1}$ and $s_{2}$ represent the maximal mating rates among female insects and, respectively, sterile and natural male insects; then $S$ measures the number of mated but no fertilized female insects with respect to the fertilized ones. Also, when $\beta=\beta_{T}, S$ measures the ratio between the number of sterile males and the number of natural insects in equilibrium. If $S$ is sufficiently high, the next generation of wild insects would be much more lower than the actual one since a considerable proportion of eggs would not hatch. If sterile male insects are sprayed for a long period of time, this pattern would drive the natural insect population to zero.

Since we are interested in solutions of biological significance, the equilibria must be adequately "positive". Then, for biological existence of $P_{2}$, condition $\alpha>\mu_{T}$ is needed, and for equilibria $P_{3-}$ and $P_{3+}$, inequalities $R>1$ and

$$
\frac{(R-1)^{2}}{4 R S} \geq 1
$$

are further required. If equality holds in (3.6) then $P_{3-}$ and $P_{3+}$ collapse to an equilibrium $P_{3}$ with $\bar{A}=\frac{(R-1)}{2 R} C$, which provides minimum $R^{*}$ that guarantees the existence of the non-trivial equilibria given by

$$
R^{*}=(1+2 S)\left[1+\sqrt{1-\frac{1}{(1+2 S)^{2}}}\right] .
$$

Notice that $R^{*}>1$.

In Table 1 we summarize the stability analysis of $P_{2}, P_{3+}$ and $P_{3-}$. The stability analysis of equilibrium points $P_{3+}$ and $P_{3-}$ is quite similar to that presented elsewhere [5]. 


\begin{tabular}{|c|l|l|l|l|}
\hline$\frac{\alpha}{\mu_{T}}$ & $R$ & existence & stable & unstable \\
\hline$<1$ & $<1$ & $P_{0}$ & $P_{0}$ & - \\
\hline$<1$ & $>1$ & $P_{0}, P_{1}$ & $P_{1}$ & $P_{0}$ \\
\hline$>1$ & $<R^{*}$ & $P_{0}, P_{1}, P_{2}$ & $P_{2}$ & $P_{0}, P_{1}$ \\
\hline$>1$ & $>R^{*}$ & $P_{0}, P_{1}, P_{2}, P_{3-}, P_{3+}$ & $P_{2}, P_{3+}$ & $P_{0}, P_{1}, P_{3-}$ \\
\hline
\end{tabular}

Table 1. Existence and stability of the equilibrium points of model (2.1).

The stability regions of equilibria can be assessed analyzing the number of immature phase in equilibrium $\bar{A}$ as a function of the basic offspring number $R$ and of the intrinsic recruitment rate $\alpha$. In this case we must analyze the range of pair of parameters $(R, \alpha)$.

Firstly, we consider the stability of equilibria with respect to $R$ when $\alpha<$ $\mu_{T}$. When $R<1$, the equilibrium $P_{0}$, where both natural and sterile populations are absent, is stable. It becomes unstable for $R>1$ and $P_{1}$ emerges as stable equilibrium. Then, the sterile population goes to extinction no matter the value of $R$, and the pest population follows its own dynamics which is governed by the basic offspring number. If $\alpha>\mu_{T}$ the sterile population approach the equilibrium $\bar{M}_{T_{1}}=\frac{\left(\alpha-\mu_{T}\right) K}{\alpha}$ (Figure 1).

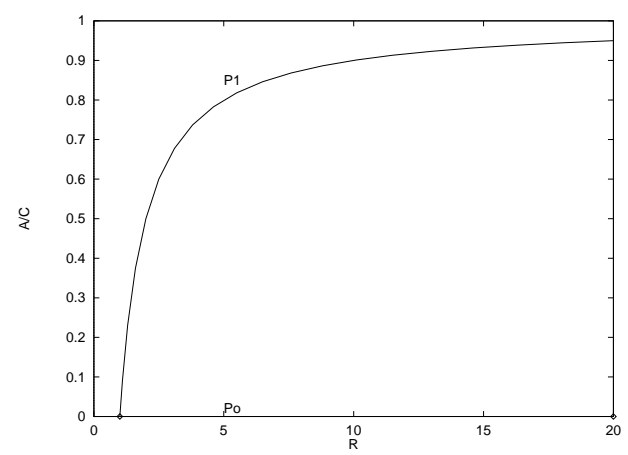

Figure 1: Bifurcation diagram of system (2.1): $A / C$ with respect to $R$, for fixed $\alpha$ with $\alpha<\mu_{T}$. At $R=1$ the equilibrium point $P_{1}$ appears, which is stable, while the equilibrium $P_{0}$ becomes unstable.

Now, we fix $R$ and increment $\alpha$ from zero to infinity. We have three cases depending on the value of $R$.

Case I. $R<1$ (Figure 2, left). $P_{0}$ is stable as long as $\alpha<\mu_{T}$ and loss its stability when $\alpha=\mu_{T}$ is reached. Then, the equilibrium $P_{2}$ emerges as stable equilibrium for $\alpha>\mu_{T}$. Here, the pest population decreases to zero since the basic offspring number is less than one, and therefore control by sterile insects is not necessary. 
Case II. $1<R<R^{*}$. The trajectories approach the unique equilibrium $P_{1}$ when $\alpha<\mu_{T}$. If $\alpha>\mu_{T}, P_{1}$ becomes unstable and $P_{2}$ is stable. Then, eradication of the pest is not achieved until the value $\alpha=\mu_{T}$ is reached.

Case III. $R>R^{*}$. Here the bifurcation diagram is illustrated in Figure 2, right. $P_{1}$ is asymptotically stable for $\alpha<\mu_{T}$, but becomes unstable and the equilibria $P_{2}, P_{3_{+}}$and $P_{3_{-}}$emerge when $\alpha>\mu_{T}$. The first two equilibria are asymptotically stable and the second one unstable. When $\alpha=\alpha^{*}$ where $\alpha^{*}$ is the value for which $(R-1)^{2} / 4 R S=1, P_{3_{+}}$and $P_{3_{-}}$collapse to the equilibrium $P_{3}$, and become unfeasible for $\alpha>\alpha^{*}$. In the last case $P_{2}$ is globally asymptotically stable. Then eradication of the pest is achieved beyond the threshold value $\alpha^{*}$. For values of $\alpha$ between $\mu_{T}$ and $\alpha^{*}$ control of the pest is not necessarily reached since the outcome will depend on the initial conditions.
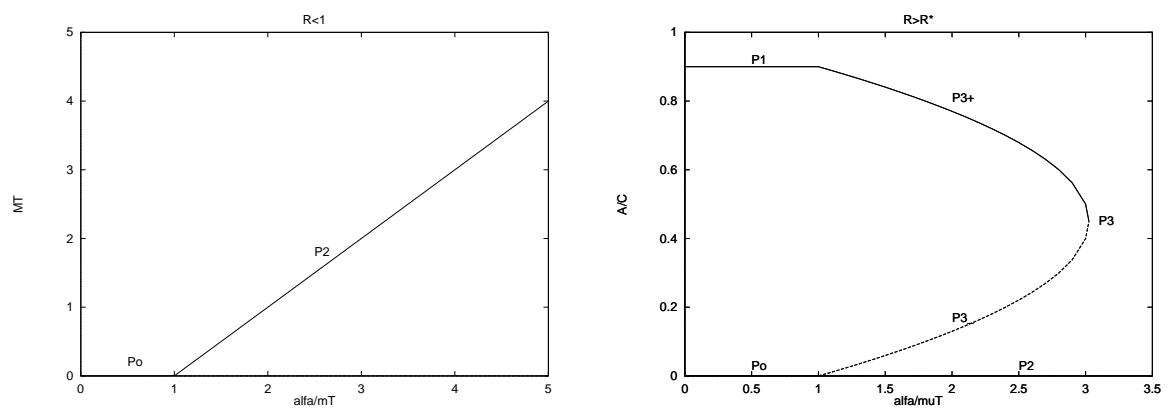

Figure 2: Bifurcation diagram of system $(2.1): M_{T}(A / C)$ with respect to $\frac{\alpha}{\mu_{T}}$, fixing $R$. When $R<1$ (left), at $\frac{\alpha}{\mu_{T}}=1$ appears $P_{2}$, which is stable, while $P_{0}$ becomes unstable. When $R>R^{*}$ (right), both $P_{0}$ and $P_{1}$ become unstable at $\frac{\alpha}{\mu_{T}}=1$, and appear $P_{3_{-}}$and $P_{3_{+}}$, which collpase to commom $P_{3}$.

\section{Conclusion}

In this paper we formulated a model to assess the effectiveness of the sterile insect release (SIT) applied to $A$. aegypti mosquito population. The results are given in terms of the basic offspring of natural population $R$, the ratio between the unfertile and fertile matings $S$, and the intrinsic rate of release of sterile males $\alpha$. The model predicts that when $R$ is above $R^{*}$, and $\alpha>\mu_{T}$, extinction of the wild insects depends on its initial population size. In this case, the use of other control strategies to reduce the population size (e.g. larvicides) before spraying are strongly recommended to guarantee the effectiveness of SIT.

For mosquito population, mating competitiveness and dispersion of sterilized males are special relevant to SIT. These properties are modelled here via the parameters $p$ and $q$. Field experiments using chemosterilized or chromosomally translocated and sex ratio-distorting $A$. aegypti have show moderately good mating competitiveness [12]. Adequated large releases (12 to 15 steriles for each wild male assuming a population with oviposition rate equal to 10 eggs per day) well mixed 
with isolated wild populations might have been expected to yield high levels of egg sterility. However, it seems that in the practice they frequently did not do so. This has been attributed to an influx of already mated females from outside the sterile-male release area [9].

Dispersal ability is a major concern for the SIT to ensure that sterile males are released near enough to all breeding sites so that they have a chance of mating with wild females. In the model the reduction of chance of encounters is modeled by the factor $q$. Thus, $q$ will have a low value for a highly dispersing species, meanwhile for a poorly dispersing one, $q \approx 1$. A. aegypti was considered a poorly dispersing species, and sterile males would need to be released at intervals of about $50 \mathrm{~m}$ along urban streets to find all the local females [13]. However, more recent studies [11] found that females can disperse over more than an $800 \mathrm{~m}$ radius. More studies should be done in order to clarify the grade of dispersion of such species.

The mathematical models presented in this paper do not include all factors affecting sterile releases. Some biological details are sacrificed in order to make models mathematical tractable. Nevertheless, according to observations, one important point that must be considered in future researches is the immigration of females that have already had fertile matings and will lay fertile eggs nullifying the effect of sterile releases. Clarification of the role of immigrants in a population is important non only for the SIT, but also for assessing whether local efforts at larval control with insecticides of environmental management could have an impact on the adult population or whether they are likely to be swamped by immigration.

Resumo. Para se controlar o mosquito transmissor da dengue, pode-se usar técnica de liberação de insetos esterilizados por radiação para deslocar insetos naturais (os que esto infestando previamente o local). Para mensurar o efeito do controle biológico, utiliza-se de modelo matemático que considera produção e liberação de machos estéreis dadas por uma logística. Os resultados mostram que fatores como forma adequada de liberação e o quanto a esterilização é eficiente devem ser cuidadosamente analisados em se tratando de mosquitos peridomésticos transmissores de dengue e febre amarela.

\section{References}

[1] H.J. Barclay, Combining methods of insect pest control. Modelling selection for resistance to control methos in combination, Reserv. Pop. Ecol., 34 (1996), 97-107.

[2] A.C. Bartlett, Insect sterility, insect genetics, and insect control. In Handbook of Pest Management in Agriculture Vol. II, pp. 279-287, D. Pimentel Ed., CRC Press, Boca Raton, FL, 1990.

[3] P.G. Coleman, L. Alphey, Editorial: Genetic control of vector population: an imminent prospect, Tropical Medicine and $\&$ International Health, 9, No. 4 (2004), 433-437.

[4] K. Dietz, The effect of immigration on genetic control, Theor. Popul. Biol., 9 (1976), 58-67. 
[5] L. Esteva, H.M. Yang, Mathematical model to assess the control of Aedes aegypti mosquitoes by the sterile insect technique, Math. Biosc., 198 (2005), 132-147.

[6] J.K. Hale, "Ordinary Differential Equations", John Wiley and Sons, New York, 1969.

[7] E.F. Knipling, Possibilities of insect control or eradication through the use of sexually sterile males, J. Econ. Entomol., 48 (1955), 459-462.

[8] E.F. Knipling, Sterile insect technique as a screwworm control measure: The concept and its development. In Symposium on Eradication of the Screwworm from the United States and Mexico, Misc. Publ. Entomol. Soc. America 62, pp. 4-7, O. H. Graham ed., College Park, MD, 1985.

[9] H. Pates, C. Curtis, Mosquito behavior and vector control, Annu. Rev. Entomol., 50 (2004), 53-70.

[10] R.E. Plant, M. Mangel, Modeling and simulation in agricultural pest management, SIAM Rev., 29 (1987), 235-261.

[11] P. Reiter, M.A. Amador, R.A. Anderson, G.C. Clark, Short report: dispersal of Aedes aegypti in an urban area after blood feeding as demonstrated by rubidium-marked eggs, Am. J. Trop. Med. Hyg., 52 (1995), 177-179.

[12] J.A. Seawright, P.E. Kaiser, D.A. Dame, Mating competitiveness of chemosterilized hybrid males of Aedes aegypti (L.) in field tests, Mosq. News, 37 (1977), 615-619.

[13] K.R.P. Singh, G.D. Brooks, Semi-automatic rlease system for distribution of mosquitoes during genetic control operations, J. Commun. Dis, 7 (1975), 288293. 